Research Article

\title{
Eg5 Overexpression Is Predictive of Poor Prognosis in Hepatocellular Carcinoma Patients
}

\author{
Can Liu, ${ }^{1}$ Nan Zhou, ${ }^{1}$ Jieying Li, ${ }^{2}$ Jun Kong, ${ }^{3}$ Xi Guan, ${ }^{3}$ and Xudong Wang ${ }^{3}$ \\ ${ }^{1}$ Department of Oncology, Affiliated Hospital of Nantong University, Nantong, Jiangsu 226001, China \\ ${ }^{2}$ Department of Pathology, Affiliated Hospital of Nantong University, Nantong, Jiangsu 226001, China \\ ${ }^{3}$ Center of Clinical Laboratory Medicine, Affiliated Hospital of Nantong University, Nantong, Jiangsu 226001, China
}

Correspondence should be addressed to Xudong Wang; wangxudong88@hotmail.com

Received 5 January 2017; Revised 25 March 2017; Accepted 19 April 2017; Published 8 June 2017

Academic Editor: Andreas Hillenbrand

Copyright (c) 2017 Can Liu et al. This is an open access article distributed under the Creative Commons Attribution License, which permits unrestricted use, distribution, and reproduction in any medium, provided the original work is properly cited.

Eg5 (kinesin spindle protein) plays an essential role in mitosis. Inhibition of Eg5 function results in cell cycle arrest at mitosis, which leads to cell death. To date, Eg5 expression and its prognostic significance have not been studied in hepatocellular carcinoma (HCC). In this study, 26 freshly frozen HCC tissue samples and matched peritumoral tissue samples were evaluated with a onestep qPCR test and immunohistochemical (IHC) analysis was conducted on 156 HCC samples to investigate the relationships among Eg5 expression, clinicopathological factors, and prognosis. Eg5 mRNA and protein expression levels were significantly higher in HCC tissues relative to matched noncancerous tissues $(p<0.05)$. High Eg5 protein expression was significantly related to liver cirrhosis $(p=0.038)$ and TNM stage $(p=0.008)$. Kaplan-Meier survival and Cox regression analyses revealed that Eg5 overexpression $(p=0.001)$, liver cirrhosis $(p=0.009)$, and TNM stage $(p=0.025)$ were independent prognostic factors for overall survival. These findings indicate that Eg5 expression can be used as a biomarker of poor prognosis and as a novel therapeutic target for HCC.

\section{Introduction}

Globally, liver cancer, especially hepatocellular carcinoma (HCC), is the sixth most frequently diagnosed cancer and shows the third highest mortality rate in cancer patients $[1,2]$. The highest incidences of HCC have been found in Asia, and increasing incidence rates have been reported in the USA and Europe [3]. Current treatments for liver cancer are varied and include surgery, targeted therapy, interventional radiology, and traditional treatment. For HCC, surgical resection remains the most common treatment, but despite operative treatment, the risk of recurrence is still high, affecting the long-term recovery of HCC patients [4]. Liver cancer has a long latency period, and most HCC patients are at an advanced stage and are often metastatic when diagnosed; for such patients, chemotherapy is a commonly used choice. Yet, conventional chemotherapy is less effective and is associated with severe side-effects $[4,5]$. Therefore, there is an urgent need to develop new therapeutic targets for HCC. So far, numberless efforts have been made to find out reliable prognostic biomarkers for HCC, and corresponding targeted drug therapy has shown an association with a favorable HCC prognosis. These markers include angiogenesis-related factors (e.g., VEGF, PDGF) $[6,7]$, epithelial growth factor receptor (EGFR) [8], and mitogen-activated protein kinase (MAPK) [9]. Therefore, it is necessary to make efforts to find further high-quality HCC prognostic markers.

Eg5 (also known as Kiff11 or kinesin spindle protein) is a member of the kinesin- 5 family and plays a crucial role in the formation and maintenance of the bipolar spindle during mitosis. Additionally, Eg5 has been regarded as an attractive target for cancer therapies [10]. Blocking Eg5 expression impairs the separation of duplicated centrosomes, leading to cell-cycle arrest in mitosis with monastrol microtubule arrays and triggering apoptotic cell death in tumor cells [11]. Indeed, recent studies have reported that Eg5 expression is associated with several malignancies, such as hepatic carcinoma [12], lung cancers [13], pancreatic cancer [14], gastric cancer [15], colorectal cancer [16], and prostate 
cancer [17]. Since the first Eg5 inhibitor, monaster, was identified in 1999 [18], a number of Eg5 inhibitors have been discovered and used in research $[16,19]$. Eg5 is overexpressed in tumors relative to normal tissues, and it is not found in postmitotic human neurons, making it an attractive target for novel anticancer therapies [20].

Although the expression of Eg5 has been associated with prognosis in certain tumor types, few studies have explored the relationship between Eg5 and liver cancer. In the present report, we studied Eg5 expression in 299 HCC samples and tumor-adjacent tissues. Furthermore, we evaluated the importance and clinical pathological significance of Eg5 in cancer patients with HCC.

\section{Materials and Methods}

2.1. HCC Sample Collection. Fresh-frozen tumor (26 HCC) and matched noncancerous tissue samples $(n=26)$ were collected from the Department of Pathology at the Hospital of Nantong University. Simultaneously, 156 formalin-fixed, paraffin-embedded HCC tumor specimens, 69 matched tumor-adjacent tissues, and 74 benign samples (cirrhosis of liver, 28 cases; liver hemangioma, 12 cases; focal nodular hyperplasia, 10 cases; intrahepatic bile duct dilatation, 9 cases; cholecystitis, 8 cases; and cyst of liver, 7 cases) were also collected from the Department of Pathology at the Hospital of Nantong University, from 2006 to 2016. Histological diagnosis of HCC was performed according to the latest WHO criteria [21]. All HCC patients were histopathologically determined by 2 pathologists. No patients received immunotherapy, chemotherapy, or radiotherapy before their surgical operation. The patient ages ranged from 31 to 79 years, with a mean age of 55 years. Survival time was defined as the interval from the date of operation to the date of death. Clinical data (including gender, age, histological type, TNM stage, $\alpha$-fetoprotein (AFP) serum level, tumor size, differentiation, and vascular invasion) and other information were retrospectively collected from each patient's records. The protocol for the present study was acquired by the Research Ethics Committee of the Affiliated Hospital of Nantong University. Twenty-six tissue blocks from patients with malignancies and with 5 years of follow-up records were also available, and these were used to construct the tissue microarray (TMA).

2.2. One-Step $q P C R$ Test. Total RNA was isolated from 26 freshly frozen HCC specimens and from matched peritumoral tissues using TRIzol reagent (Invitrogen, USA). Immediately after extraction, the RNA quantity and quality were analyzed by spectrophotometry. The RNA was then reverse-transcribed into cDNA with Moloney murine leukemia virus reverse transcriptase (Promega, USA). One-step PCR was performed as previously reported [22]. Eg5specific oligonucleotide primers (forward 5'-GAA CAA TCA TTA GCA GCA GAA-3' and reverse 5'-TCA GTA TAG ACA CCA CAG TTG-3') were designed to yield a 118 bp PCR product. Eg5 gene expression was standardized against $\beta$-actin expression (forward primer $5^{\prime}$-TAA TCT TCG CCT TAA TAC TT- $3^{\prime}$ and reverse primer $5^{\prime}$-AGC
CTT CAT ACA TCT CAA-3') (Supplementary Table available online at https://doi.org/10.1155/2017/2176460). The liver cancer of TCGA data was downloaded from https:// genome-cancer.ucsc.edu/proj/site/hgHeatmap/.

2.3. Immunohistochemical (IHC) Staining. A total of 156 HCC tissue samples, 74 matched adjacent liver tissue samples, and 69 benign tissue samples were collected and used in this study. TMAs were assembled using a tissue array instrument (Quick-Ray, UT06, UNITMA, Korea). All tissues were formalin-fixed and paraffin-embedded, and the TMA samples were cut into $4 \mu \mathrm{m}$ sections and placed on Superfrost charged glass microscope slides.

The TMA microarray sections were separately stained with anti-Eg5 antibody (1:100; Abcam, USA) overnight at $4^{\circ} \mathrm{C}$, washed with PBS, incubated with a biotinylated secondary antibody for $15 \mathrm{~min}$ and washed again. The sections were stained with 3,3-diaminobenzidine (DAB) chromogen solution for $15 \mathrm{~min}$, and the sections were then counterstained with hematoxylin. For negative controls, PBS was used in place of the primary antibody. In the present study, EG5 immunostaining for each slide was scored by two independent pathologists according to the staining intensity and the percentage of EG5-positive cells at that intensity. Percentage of Eg5 gene-positive cells were scored as follows: 0 (0\% tumor cells stained), 1 (1-33\% tumor cells stained), 2 (34-66\% tumor cells stained), and 3 (67-100\% tumor cells stained). Additionally, the Eg5 staining intensities were scored as follows: 0 (+, negative), 1 (+, weakly positive), $2(++$, moderately positive), and $3(+++$, strongly positive). The final EG5 gene staining score was the sum of the intensity and percentage scores, and samples with a summed score of less than 2 were defined as exhibiting low expression, while those with a summed score of 3-6 were defined as exhibiting high expression $[23,24]$.

2.4. Statistical Methods. The Eg5 mRNA expression levels in the freshly frozen HCC and matched normal tissues were analyzed using the nonparametric Wilcoxon signed-rank test. The relationships between Eg5 expression levels in the tumor samples and patient clinicopathological features were evaluated using the $\chi^{2}$ test. The survival rate was calculated with the Kaplan-Meier and log-rank tests. The TCGA data for liver cancer was downloaded from https://cancergenome.ucsc.edu. Univariate and multivariate analyses were performed using the Cox hazard regression method. $p$ values of less than 0.05 were considered to be statistically significant. Data were analyzed using STATA 12.0 software (Stata Corporation, USA).

\section{Results}

3.1. Eg5 mRNA Expression in HCC and Peritumoral Tissues. To investigate Eg5 mRNA expression in HCC patients, RNA was extracted from 26 cancerous and 26 adjacent tissues and analyzed using one-step qPCR. When comparing Eg5 expression in cancerous and noncancerous tissues, we found that Eg5 mRNA expression was significantly higher in the HCC tissues than in the normal tissues 
TCGA dataset (LIHC)

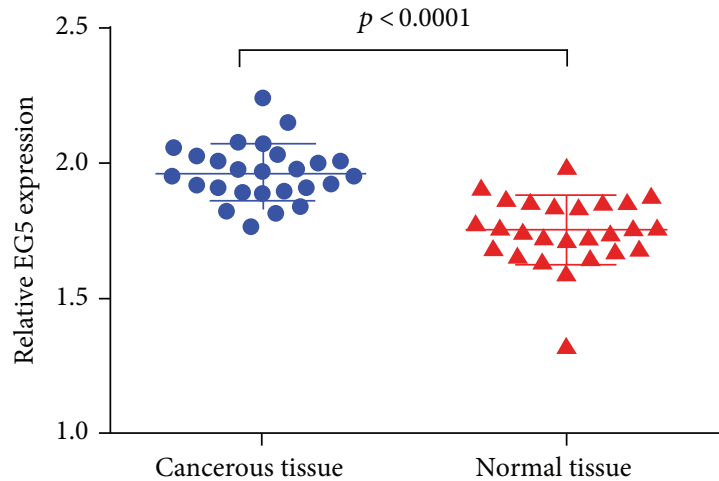

(a)

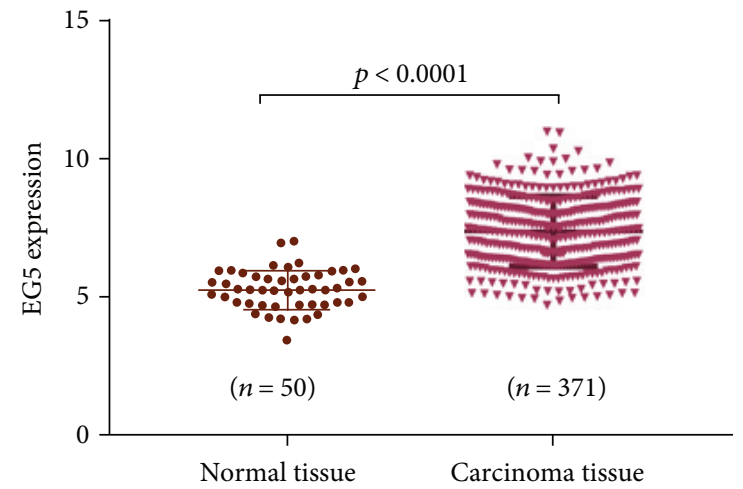

(b)

FIGURE 1: Eg5 mRNA expression in HCC and normal tissues. (a) One-step qPCR was performed to compare Eg5 mRNA expression levels in cancerous tissues with those in adjacent normal tissues. The Eg5 mRNA level in the HCC tissue (1.976 \pm 0.02068$)$ was higher on average than that in the matched adjacent tissue. $\beta$-Actin was used as an internal control. (b) In TCGA dataset, the Eg5 mRNA level in the carcinoma tissue was higher on average than that in the matched normal tissue $(p<0.0001)$.

$(1.967 \pm 0.020$ versus $1.754 \pm 0.024$, resp.; $t=6.559$, $p<0.0001$ ) (Figure 1(a)). And in TCGA dataset, 371 cancerous tissues and 50 noncancerous liver tissues were detected using HiSeq; the expression of Eg5 mRNA in normal and carcinoma tissues has significant statistical differences $(p<0.0001)$ (Figure 1(b)).

3.2. Eg5 Protein Expression in HCC Tissues Detected by IHC. We studied Eg5 expression in the HCC patient samples using IHC with a TMA (156 HCC samples, 69 matched peritumoral samples, and 74 benign liver cancer tissue samples). Typical IHC staining patterns for Eg5 are shown in Figure 2. Positive staining for Eg5 was predominantly localized to the cytoplasm of HCC cells; however, in some samples, positive Eg5 staining was also observed at the cell membranes. The results showed that Eg5 expression was markedly high in the HCC tumors, but low or negative in benign or adjacent liver tissue (Figure 2). Among the HCC tumors, high cytoplasmic expression of Eg5 was found in $67.31 \%(105 / 156)$ of the liver cancer samples, compared with only $43.48 \%(30 / 69)$ of benign tissues and $47.30 \%$ $(35 / 74)$ of peritumoral tissues. We determined the cutoff point for Eg5 to be 150 using the X-tile software program (the Rimm Lab at Yale University; http://medicine.yale.edu/ lab/rimm/research/software.aspx), and the data represented statistical significance $\left(\chi^{2}=14.738, p<0.001\right)$ (Table 1$)$.

\subsection{Relationship between Eg5 Expression and} Clinicopathological Features in HCC Samples. To confirm the important role of Eg5 in liver cancer progression, we summarized the relationship between Eg5 expression and the major clinicopathological variables of HCC in Table 2. Stratifying the clinical parameters by the two Eg5 expression levels, we found that Eg5 expression was significantly associated with TNM stage $(p=0.008)$ and liver cirrhosis $(p=0.038)$ (Table 2). However, we found no statistically significant association between high Eg5 expression and age, gender, liver cirrhosis, differentiation, gross classification, hepatitis B virus infection, vascular invasion, or AFP levels (Table 2).

3.4. Eg5 Overexpression Predicts Poorer Prognosis. Using univariate Cox regression analysis, we found that high Eg5 expression was significantly associated with inferior outcome in HCC patients $(p=0.001)$. Additionally, other HCC clinical prognostic factors, such as liver cirrhosis $(p=0.003)$, gross classification $(p=0.007)$, and TNM stage $(p=0.011)$, were significantly correlated with a decreased 5-year survival rate (Table 3). Factors that were independent of prognostic factors in univariate models were assessed in the multivariate Cox regression analysis. Moreover, our data showed that high Eg5 expression $(p=0.001)$, liver cirrhosis $(p=0.009)$, and TNM stage $(p=0.025)$ were all significant predictors of survival in HCC. Kaplan-Meier survival analysis also demonstrated that HCC patients with high Eg5 expression, advanced TNM stage, or liver cirrhosis had significantly shorter survival times (Figures 3(a), 3(b), and 3(c)). Simultaneously, in TCGA dataset, patients with low Eg5 expression levels exhibited higher overall survival rates than patients with high Eg5 expression levels (Figure 3(d)).

\section{Discussion}

Eg5, a microtubule-based motor of the kinesin family, is considered to have a key role in the formation and maintenance of the bipolar spindle during mitosis and has been regarded as a key target of anticancer treatment $[10,25,26]$. In addition to its role in mitosis, an increasing number of studies have revealed other possible biological functions of Eg5. Liu et al. reported that $\mathrm{Eg} 5$ is involved in an immune regulation process; Eg5 interacts with Tat, which blocks cell-cycle progression in CD4-positive T lymphocytes, thereby leading to apoptosis [27]. Groth-Pedersen et al. observed that Eg5 siRNAs inhibit lysosomal trafficking and induce lysosomal membrane permeabilization by increasing lysosomal volume [28]. Additionally, Eg5 also plays important roles in protein 


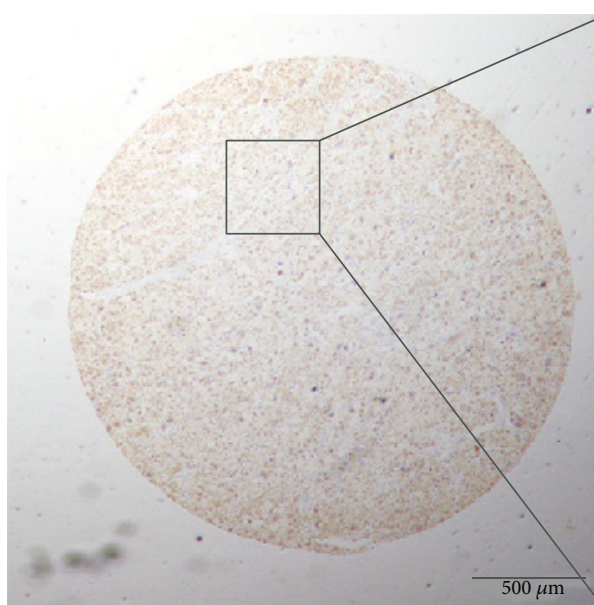

(a)

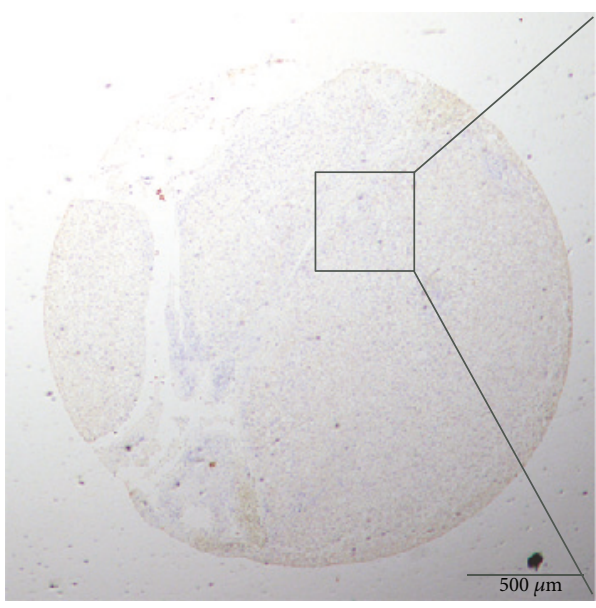

(c)

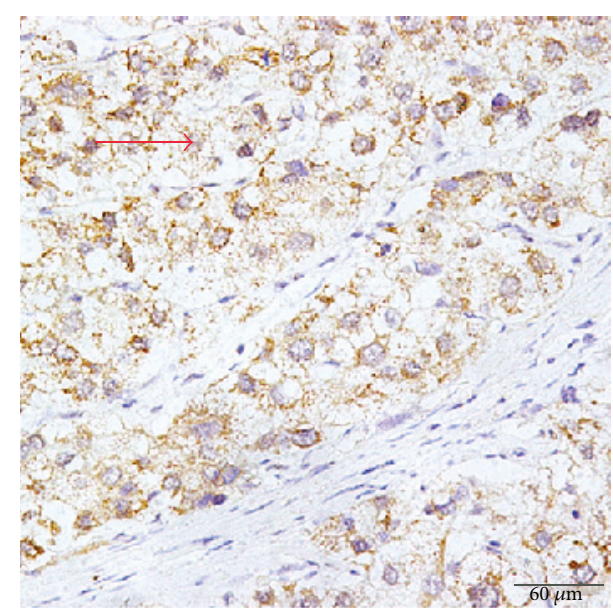

(b)

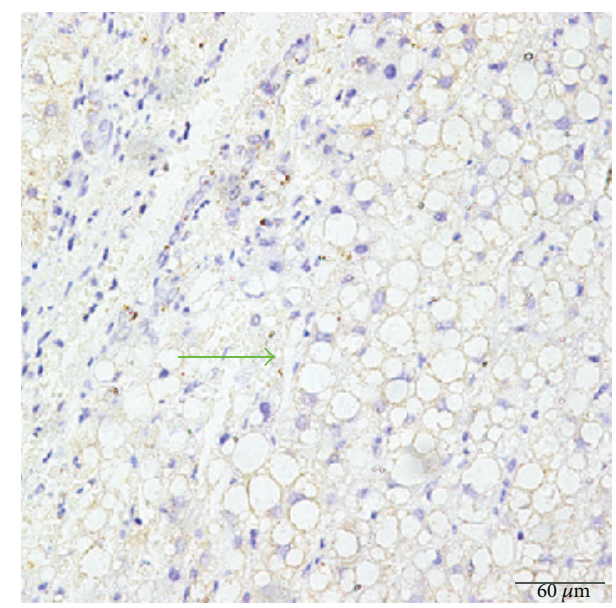

(d)

FIgURE 2: Immunohistochemical (IHC) staining for Eg5 expression in cancerous and peritumoral tissues. (a, b) Positive cytoplasmic IHC staining (red arrow) for Eg5 in HCC tissue samples. (c, d) Negative IHC staining (green arrow) for Eg5 in normal tissue samples (original magnification: $\times 40$ in (a) and (c); $\times 400$ in (b) and (d)).

TABLE 1: Eg5 IHC staining in benign, cancerous, and adjacent liver tissues.

\begin{tabular}{lcccc}
\hline \multicolumn{1}{c}{ Tissue sample } & $n$ & \multicolumn{2}{c}{ Eg5 expression, $n(\%)$} & Pearson $\chi^{2}$ \\
& & Low or none & Positive & \\
Liver tissue with benign disease & 74 & $39(52.70)$ & $35(47.30)$ & 14.738 \\
Adjacent liver tissue & 69 & $39(56.52)$ & $30(43.48)$ & $0.001^{*}$ \\
Liver cancer & 156 & $51(32.69)$ & $105(67.31)$ & \\
\hline
\end{tabular}

${ }^{*} p<0.05$.

translation, neuronal survival, cancer cell migration, and angiogenesis impairment [29-33]; therefore, Eg5 is believed to be a potential therapeutic target for solid tumors. High Eg5 expression has been reported in different types of malignancies [12-17]. However, to our knowledge, associations between Eg5 expression level and clinical parameters in HCC to evaluate the significant clinicopathological role of Eg5 have not yet been investigated.

In the present study, the qRT-PCR data showed that the mean Eg5 mRNA expression level in cancerous tissue was higher than that in the corresponding normal tissue, in accord with the results of IHC analysis in HCC samples. TMA and IHC analyses of the HCC tissues showed that the high Eg5 protein expression was mainly observed in the tumor cell cytoplasm, in agreement with previous reports [23]. Positive Eg5 protein staining was indicated in $67.31 \%$ $(105 / 156)$ of the HCC tissue samples, representing an increase over the adjacent liver and benign liver disease tissues. These results verified our hypothesis and were in accord with previous reports indicating that high Eg5 protein 
TABle 2: Patient clinicopathological characteristics according to the Eg5 score.

\begin{tabular}{|c|c|c|c|c|c|}
\hline \multirow{2}{*}{ Characteristic } & \multirow{2}{*}{$n$} & \multicolumn{2}{|c|}{ Eg5 expression, $n(\%)$} & \multirow{2}{*}{ Pearson $\chi^{2}$} & \multirow[b]{2}{*}{$p$} \\
\hline & & Negative & Positive & & \\
\hline Total & 156 & $51(32.69)$ & $105(67.31)$ & & \\
\hline Gender & & & & 0.132 & 0.717 \\
\hline Male & 119 & $38(31.93)$ & $81(68.07)$ & & \\
\hline Female & 37 & $13(35.14)$ & $24(64.86)$ & & \\
\hline Age & & & & 1.867 & 0.172 \\
\hline$<60$ & 109 & $39(35.78)$ & $70(64.22)$ & & \\
\hline$\geq 60$ & 45 & $11(24.44)$ & $34(75.56)$ & & \\
\hline Unknown & 2 & & & & \\
\hline Differentiation & & & & 2.092 & 0.351 \\
\hline Well & 12 & $6(50.00)$ & $6(50.00)$ & & \\
\hline Middle & 113 & $34(30.09)$ & $79(69.91)$ & & \\
\hline Poor & 31 & $11(35.48)$ & $20(64.52)$ & & \\
\hline Liver cirrhosis & & & & 4.298 & $0.038^{*}$ \\
\hline Yes & 99 & $26(26.26)$ & $73(73.74)$ & & \\
\hline No & 46 & $20(43.48)$ & $26(56.52)$ & & \\
\hline Unknown & 11 & & & & \\
\hline Gross classification & & & & 0.093 & 0.760 \\
\hline Solitary & 139 & $46(33.09)$ & $93(66.91)$ & & \\
\hline Multiple & 17 & $5(29.41)$ & $12(70.59)$ & & \\
\hline Hepatitis B viral infection & & & & 1.115 & 0.291 \\
\hline Yes & 12 & $2(16.67)$ & $10(83.33)$ & & \\
\hline No & 128 & $39(30.47)$ & $89(69.53)$ & & \\
\hline Unknown & 16 & & & & \\
\hline Vascular invasion & & & & 2.121 & 0.145 \\
\hline Yes & 68 & $18(26.47)$ & $50(73.53)$ & & \\
\hline No & 88 & $33(37.50)$ & $55(62.50)$ & & \\
\hline Tumor diameter & & & & 1.974 & 0.160 \\
\hline$<5 \mathrm{~cm}$ & 93 & $35(37.63)$ & $58(62.37)$ & & \\
\hline$\geq 5 \mathrm{~cm}$ & 60 & $16(26.67)$ & $44(73.33)$ & & \\
\hline Unknown & 3 & & & & \\
\hline TNM stage & & & & 9.710 & $0.008^{*}$ \\
\hline I & 79 & $30(37.97)$ & $49(62.03)$ & & \\
\hline II & 66 & $21(31.82)$ & $45(68.18)$ & & \\
\hline III & 11 & $0(0.00)$ & $11(100.00)$ & & \\
\hline$\alpha$-Fetoprotein $(\mathrm{ng} / \mathrm{ml})$ & & & & 0.959 & 0.327 \\
\hline$<20$ & 51 & $13(25.49)$ & $38(74.51)$ & & \\
\hline$\geq 20$ & 68 & $23(33.82)$ & $45(66.18)$ & & \\
\hline Unknown & 37 & & & & \\
\hline
\end{tabular}

expression levels are present in cancer tissue samples, such as in $60.58 \%$ of laryngeal squamous cell carcinoma [23] and $51.8 \%$ of renal cell carcinoma (RCC) [34]. Differences among studies may be due to the cutoff points that were chosen. Overall, the results support the conclusion that Eg5 expression levels in HCC cells are higher than those in normal hepatic cells. However, in our study, the expression of Eg5 in benign samples is relatively higher $(47.30 \%$, $35 / 74$ ); this result might be due to 74 cases of benign tissues containing 28 cases of cirrhosis, 12 cases of liver hemangioma, and 10 cases of focal nodular hyperplasia. In the histopathology, liver cirrhosis has hepatocellular nodular regeneration and connective tissue hyperplasia; hepatic hemangioma with vascular endothelial abnormalities and focal nodular hyperplasia have fibrous tissue and small bile duct hyperplasia. Moreover, it may be that, in the present study, the number of benign samples is relatively insufficient.

We discovered a correlation between Eg5 expression, clinicopathological parameters, and prognosis. High Eg5 
TABLE 3: Univariate and multivariate analyses of prognostic factors for overall survival in liver cancer patients.

\begin{tabular}{|c|c|c|c|c|c|c|c|c|}
\hline \multirow{3}{*}{ Eg5 expression } & \multicolumn{4}{|c|}{ Univariate analysis } & \multicolumn{4}{|c|}{ Multivariate analysis } \\
\hline & \multirow[t]{2}{*}{ HR } & \multirow[t]{2}{*}{$p>|z|$} & \multicolumn{2}{|c|}{$95 \% \mathrm{CI}$} & \multirow[t]{2}{*}{$\mathrm{HR}$} & \multirow[t]{2}{*}{$p>|z|$} & \multicolumn{2}{|c|}{$95 \% \mathrm{CI}$} \\
\hline & & & & & & & & \\
\hline High versus low & 4.490 & $<0.001^{*}$ & 2.511 & 8.030 & 3.913 & $<0.001^{*}$ & 2.136 & 7.167 \\
\hline \multicolumn{9}{|l|}{ Age (years) } \\
\hline$\leq 60$ versus $>60$ & 0.983 & 0.949 & 0.589 & 1.643 & & & & \\
\hline \multicolumn{9}{|l|}{ Gender } \\
\hline Male versus female & 0755 & 0.260 & 0.463 & 1.323 & & & & \\
\hline \multicolumn{9}{|l|}{ Liver cirrhosis } \\
\hline Yes versus no & 2.396 & $0.003^{*}$ & 1.339 & 4.286 & 2.169 & $0.009^{*}$ & 1.210 & 3.889 \\
\hline \multicolumn{9}{|l|}{ Gross classification } \\
\hline Solitary versus multiple & 2.292 & $0.007^{*}$ & 1.254 & 4.188 & & & & \\
\hline \multicolumn{9}{|l|}{ Differentiation } \\
\hline Well versus middle versus poor & 1.217 & 0.353 & 0.804 & 1.843 & & & & \\
\hline \multicolumn{9}{|l|}{ Hepatitis B viral infection } \\
\hline Yes versus no & 1.489 & 0.438 & 0.544 & 4.072 & & & & \\
\hline \multicolumn{9}{|l|}{ Vascular invasion } \\
\hline Yes versus no & 1.132 & 0.582 & 0.728 & 1.761 & & & & \\
\hline \multicolumn{9}{|l|}{ Tumor diameter $(\mathrm{cm})$} \\
\hline$<5$ versus $\geq 5$ & 1.287 & 0.277 & 0.816 & 2.029 & & & & \\
\hline \multicolumn{9}{|l|}{ TNM stage } \\
\hline I versus II versus III & 1.604 & $0.011^{*}$ & 1.112 & 2.313 & 1.524 & $0.025^{*}$ & 1.053 & 2.206 \\
\hline \multicolumn{9}{|l|}{ Preoperative $\alpha$-fetoprotein (ng/ml) } \\
\hline$<20$ versus $\geq 20$ & 0.900 & 0.658 & 0.564 & 1.436 & & & & \\
\hline
\end{tabular}

${ }^{*} p<0.05$; HR: hazard ratio; $95 \%$ CI: $95 \%$ confidence interval.

expression was correlated with TNM stage $(p=0.008)$; this result verified the pro-oncogenic role of $\mathrm{Eg} 5$ and is in agreement with reports that suggested a tumorigenic role in malignancies $[23,34,35]$. Additionally, we found the overexpression of Eg5 is related to liver cirrhosis $(p=0.038)$. EG5 is a microtubule-based motor of the kinesin family; it has a key role in the formation and maintenance of the bipolar spindle during mitosis. Therefore, the result may be owning to cirrhosis of the liver with extensive residual hepatocellular nodular regeneration and connective tissue hyperplasia. Furthermore, univariate and multivariate analyses indicated that increased Eg5 expression, advanced TNM stage, and liver cirrhosis are independent factors for unfavorable overall survival of HCC patients. Finally, our data clearly suggest that high Eg5 expression is significantly associated with poor HCC outcomes.

Interestingly, Oncomine data suggested that Eg5 transcripts are not overexpressed in kidney cancer. However, recent evidence has shown that, compared to that in normal tissue, higher Eg5 expression in RCC tumors leads to poor disease outcomes [34], consistent with the results of our study. Additionally, data from a lung cancer study showed that patients with high Eg5 expression had a higher response rate to chemotherapy than patients with low Eg5 expression (37\% versus $10 \%$ ) [13]; this opposite result might be due to differences in tumor types or in the internal environments of the patients. Moreover, Eg5 might possess multiple roles in cancer. A previous research study showed that SHIP2 (SH2-containing inositol 5-phosphatase 2) possesses both anticancer activities and oncogenic functions [36]. Therefore, our research requires further validation with a larger sample size, and we should perform in vitro and in vivo studies to verify the roles of Eg5 in HCC development.

The present study is the first to demonstrate the important role for Eg5 in HCC, not only at the mRNA level but also at the protein level. Our results provide evidence that Eg5 overexpression is related to poorer survival in HCC patients, which may be helpful in future research studies aimed at understanding the molecular mechanisms that underlie the development of HCC. Our findings have established the potential clinical value of Eg5 as a novel biomarker in HCC, and targeting Eg5 might provide a novel strategy for HCC treatment.

\section{Conclusions}

The expression of Eg5 in HCC was markedly higher when compared to that in the matched noncancerous tissues, and this overexpression was significantly related to liver cirrhosis $(p=0.038)$, advanced TNM stage $(p=0.008)$, and a poor prognosis. Overall, these findings suggest that Eg5 expression can be used as a biomarker of poor prognosis and even a novel therapeutic target for HCC. 

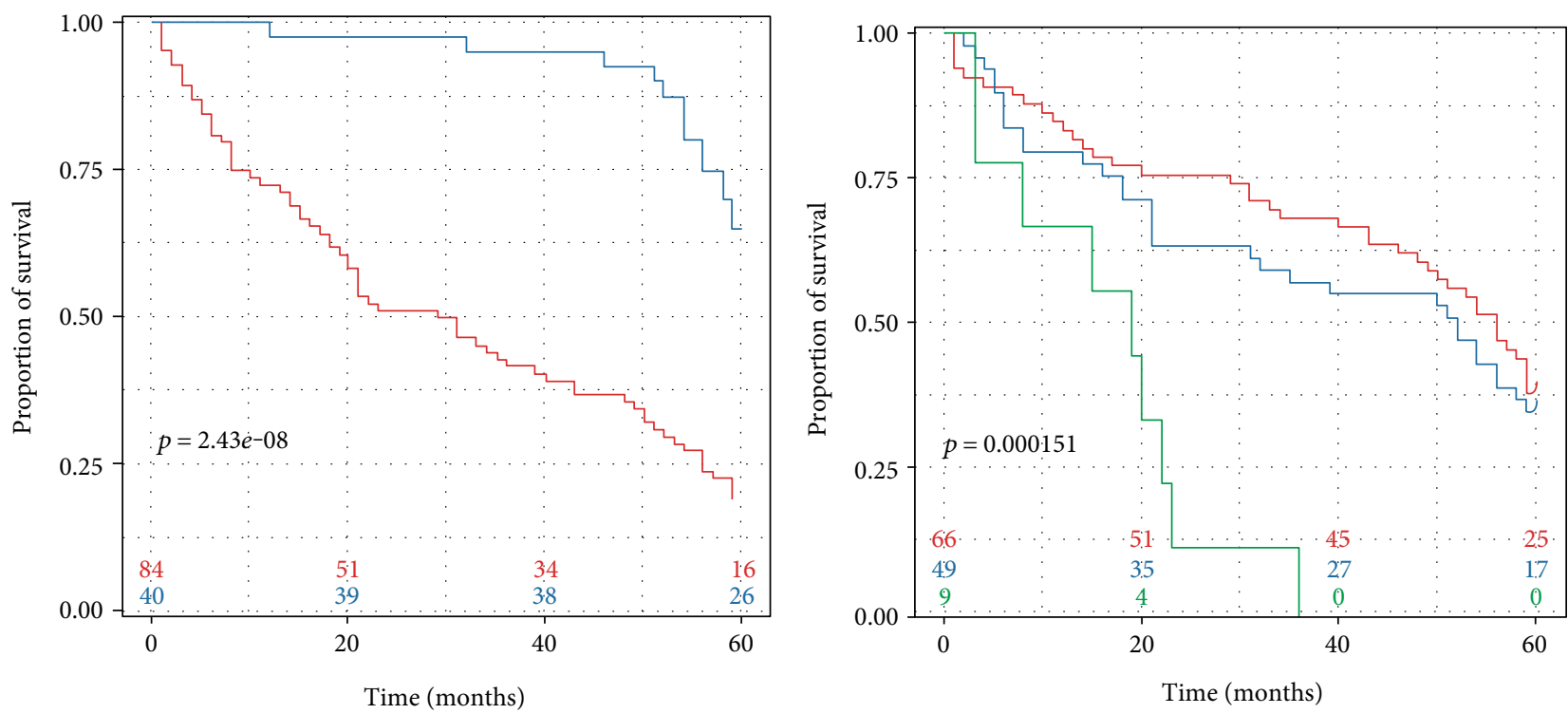

Eg5_expression

$\uparrow$ High

+ Low

TNM_stage

$+\mathrm{I}$

+ II

+ III-IV

(a)

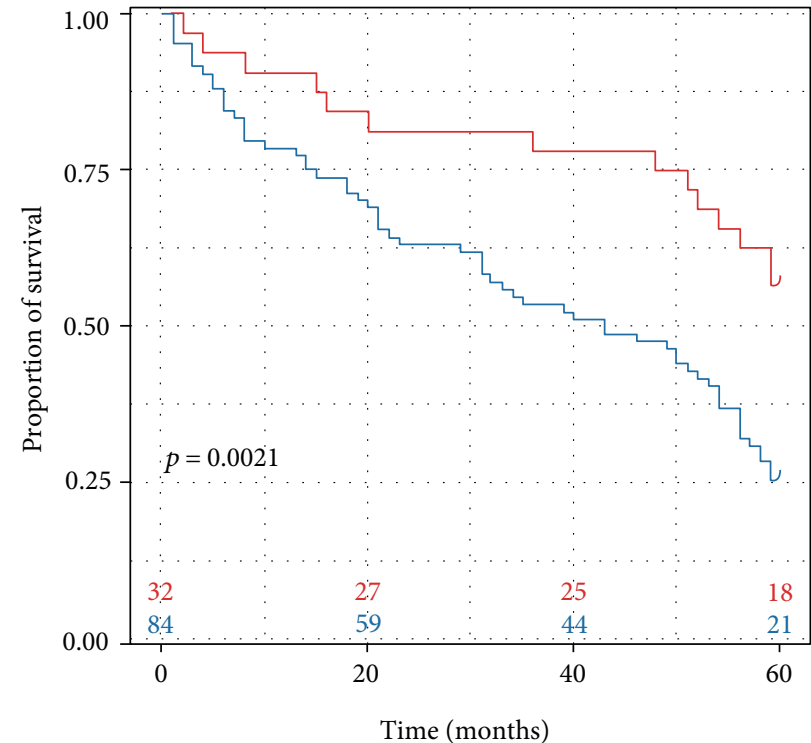

Liver_cirrhosis

+ No

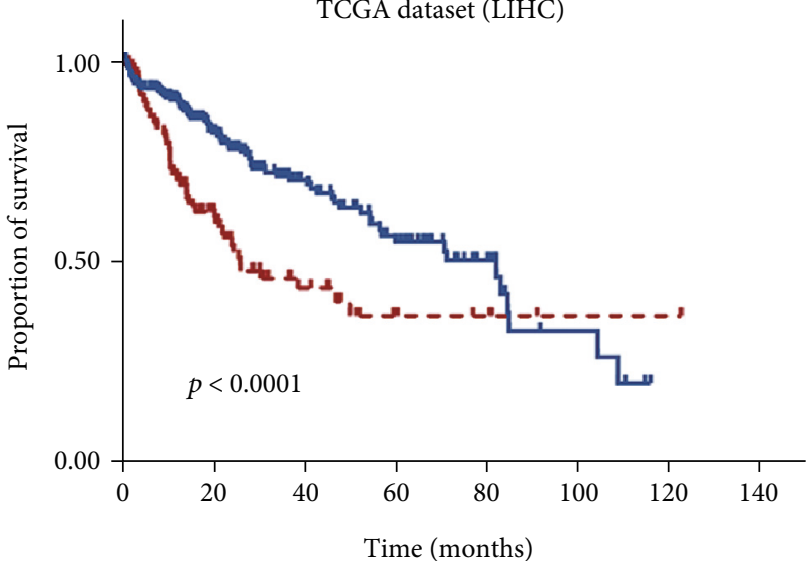

+ Yes

Low Eg5 expression $(n=252)$

- - - High Eg5 expression $(n=108)$

(c)

(d)

Figure 3: Kaplan-Meier survival curves of HCC patients after surgical therapy. (a) Patients with high Eg5 expression levels (red line) exhibited lower overall survival rates than patients with low Eg5 expression levels (blue line). (b) Patients at an advanced TNM stage (stages III-IV) (green line) exhibited a statistically lower overall survival rate than those at an early TNM stage. (c) Patients with liver cirrhosis (blue line) exhibited poor prognoses compared with patients without liver cirrhosis. (d) In TCGA dataset, patients with high Eg5 expression levels (red line) exhibited lower overall survival rates than patients with low Eg5 expression levels (blue line). 


\section{Conflicts of Interest}

The authors declare that there are no competing financial interests.

\section{Acknowledgments}

This study was supported by the fifth batch of "226 Talent Program" from Nantong City, Jiangsu, China; Medical Talent Program from the Affiliated Hospital of Nantong University, China; and Start Program for Oversea Returnee from Department of Jiangsu Human Resources and Social Security. The authors are grateful to Jianfei Huang for his assistance with statistics.

\section{References}

[1] J. Ferlay, I. Soerjomataram, R. Dikshit et al., "Cancer incidence and mortality worldwide: sources, methods and major patterns in GLOBOCAN 2012," International Journal of Cancer, vol. 136, no. 5, pp. E359-E386, 2015.

[2] K. Okuda, "Hepatocellular carcinoma," Journal of Hepatology, vol. 32, 1 Supplement, pp. 225-237, 2000.

[3] S. Mittal and H. B. El-Serag, "Epidemiology of hepatocellular carcinoma: consider the population," Journal of Clinical Gastroenterology, vol. 47, Supplement 1, pp. S2-S6, 2013.

[4] S. Lin, K. Hoffmann, and P. Schemmer, "Treatment of hepatocellular carcinoma: a systematic review," Liver Cancer, vol. 1, no. 3-4, pp. 144-158, 2012.

[5] M. Mendizabal and K. R. Reddy, "Current management of hepatocellular carcinoma," The Medical Clinics of North America, vol. 93, no. 4, pp. 885-900, 2009.

[6] M. W. Welker and J. Trojan, "Anti-angiogenesis in hepatocellular carcinoma treatment: current evidence and future perspectives," World Journal of Gastroenterology, vol. 17, no. 26, pp. 3075-3081, 2011.

[7] G. M. Keating, "Sorafenib: a review in hepatocellular carcinoma," Targeted Oncology, vol. 12, no. 2, pp. 243-253, 2017.

[8] Y. J. Chen, C. W. Chi, W. C. Su, and H. L. Huang, "Lapatinib induces autophagic cell death and inhibits growth of human hepatocellular carcinoma," Oncotarget, vol. 5, no. 13, pp. 4845-4854, 2014

[9] S. Tanaka and S. Arii, "Molecular targeted therapies in hepatocellular carcinoma," Seminars in Oncology, vol. 39, no. 4, pp. 486-492, 2012.

[10] L. C. Kapitein, E. J. Peterman, B. H. Kwok, J. H. Kim, T. M. Kapoor, and C. F. Schmidt, "The bipolar mitotic kinesin Eg5 moves on both microtubules that it crosslinks," Nature, vol. 435, no. 7038, pp. 114-118, 2005.

[11] M. Shimizu, H. Ishii, N. Ogo et al., "S-trityl-L-cysteine derivative induces caspase-independent cell death in K562 human chronic myeloid leukemia cell line," Cancer Letters, vol. 298, no. 1, pp. 99-106, 2010.

[12] L. Yang, C. Jiang, F. Liu, Q. D. You, and W. T. Wu, "Cloning, enzyme characterization of recombinant human Eg5 and the development of a new inhibitor," Biological \& Pharmaceutical Bulletin, vol. 31, no. 7, pp. 1397-1402, 2008.

[13] T. Saijo, G. Ishii, A. Ochiai et al., "Eg5 expression is closely correlated with the response of advanced non-small cell lung cancer to antimitotic agents combined with platinum chemotherapy," Lung Cancer, vol. 54, no. 2, pp. 217-225, 2006.
[14] M. Liu, X. Wang, Y. Yang et al., "Ectopic expression of the microtubule-dependent motor protein Eg5 promotes pancreatic tumourigenesis," The Journal of Pathology, vol. 221, no. 2, pp. 221-228, 2010.

[15] G. R. Yan, F. Y. Zou, B. L. Dang et al., "Genistein-induced mitotic arrest of gastric cancer cells by downregulating KIF20A, a proteomics study," Proteomics, vol. 12, no. 14, pp. 2391-2399, 2012.

[16] R. Nakai, S. Iida, T. Takahashi et al., "K858, a novel inhibitor of mitotic kinesin Eg5 and antitumor agent, induces cell death in cancer cells," Cancer Research, vol. 69, no. 9, pp. 3901-3909, 2009.

[17] M. D. Wissing, E. S. De Morree, V. O. Dezentje et al., "Nuclear Eg5 (kinesin spindle protein) expression predicts docetaxel response and prostate cancer aggressiveness," Oncotarget, vol. 5, no. 17, pp. 7357-7367, 2014.

[18] T. U. Mayer, T. M. Kapoor, S. J. Haggarty, R. W. King, S. L. Schreiber, and T. J. Mitchison, "Small molecule inhibitor of mitotic spindle bipolarity identified in a phenotype-based screen," Science, vol. 286, no. 5441, pp. 971-974, 1999.

[19] S. Ding, K. Nishizawa, T. Kobayashi et al., "A potent chemotherapeutic strategy for bladder cancer: (S)-methoxy-trityl-Lcystein, a novel Eg5 inhibitor," The Journal of Urology, vol. 184, no. 3, pp. 1175-1181, 2010.

[20] W. Tao, V. J. South, R. E. Diehl et al., "An inhibitor of the kinesin spindle protein activates the intrinsic apoptotic pathway independently of p53 and de novo protein synthesis," Molecular and Cellular Biology, vol. 27, no. 2, pp. 689-698, 2007.

[21] S. R. Hamilton and L. A. Aaltonen, "Pathology and genetics of tumours of the digestive system," in World Health Organization Classification of Tumours (WHO OMS), pp. 157-172, IARC Press, Lyon, 2000.

[22] J. Huang, H. Zhu, X. Wang et al., "The patterns and expression of KDR in normal tissues of human internal organs," Journal of Molecular Histology, vol. 42, no. 6, pp. 597-603, 2011.

[23] M. Lu, H. Zhu, X. Wang et al., "The prognostic role of Eg5 expression in laryngeal squamous cell carcinoma," Pathology, vol. 48, no. 3, pp. 214-218, 2016.

[24] J. Huang, X. Zhang, Q. Tang et al., "Prognostic significance and potential therapeutic target of VEGFR2 in hepatocellular carcinoma," Journal of Clinical Pathology, vol. 64, no. 4, pp. 343-348, 2011.

[25] K. W. Wood, W. D. Cornwell, and J. R. Jackson, "Past and future of the mitotic spindle as an oncology target," Current Opinion in Pharmacology, vol. 1, no. 4, pp. 370-377, 2001.

[26] X. Liu, H. Gong, and K. Huang, "Oncogenic role of kinesin proteins and targeting kinesin therapy," Cancer Science, vol. 104, no. 6, pp. 651-656, 2013.

[27] M. Liu, D. Li, L. Sun et al., "Modulation of Eg5 activity contributes to mitotic spindle checkpoint activation and Tat-mediated apoptosis in CD4-positive T-lymphocytes," The Journal of Pathology, vol. 233, no. 2, pp. 138-147, 2014.

[28] L. Groth-Pedersen, S. Aits, E. Corcelle-Termeau, N. H. Petersen, J. Nylandsted, and M. Jaattela, "Identification of cytoskeleton-associated proteins essential for lysosomal stability and survival of human cancer cells," PloS One, vol. 7, no. 10, article e45381, 2012.

[29] K. M. Bartoli, J. Jakovljevic, J. J. Woolford, and W. S. Saunders, "Kinesin molecular motor Eg5 functions during polypeptide synthesis," Molecular Biology of the Cell, vol. 22, no. 18, pp. 3420-3430, 2011. 
[30] R. Midorikawa, Y. Takei, and N. Hirokawa, "KIF4 motor regulates activity-dependent neuronal survival by suppressing PARP-1 enzymatic activity," Cell, vol. 125, no. 2, pp. 371383, 2006.

[31] A. Falnikar, S. Tole, and P. W. Baas, "Kinesin-5, a mitotic microtubule-associated motor protein, modulates neuronal migration," Molecular Biology of the Cell, vol. 22, no. 9, pp. 1561-1574, 2011.

[32] X. D. Sun, X. J. Shi, X. O. Sun et al., "Dimethylenastron suppresses human pancreatic cancer cell migration and invasion in vitro via allosteric inhibition of mitotic kinesin Eg5," Acta Pharmacologica Sinica, vol. 32, no. 12, pp. 1543-1548, 2011.

[33] P. Exertier, S. Javerzat, B. Wang et al., "Impaired angiogenesis and tumor development by inhibition of the mitotic kinesin Eg5," Oncotarget, vol. 4, no. 12, pp. 2302-2316, 2013.

[34] D. Sun, J. Lu, K. Ding et al., “The expression of Eg5 predicts a poor outcome for patients with renal cell carcinoma," Medical Oncology, vol. 30, no. 1, p. 476, 2013.

[35] S. Ding, N. Xing, J. Lu et al., "Overexpression of Eg5 predicts unfavorable prognosis in non-muscle invasive bladder urothelial carcinoma," International Journal of Urology, vol. 18, no. 6, pp. 432-438, 2011.

[36] J. Yang, M. Fu, Y. Ding et al., "High SHIP2 expression indicates poor survival in colorectal cancer," Disease Markers, vol. 2014, Article ID 218968, 7 pages, 2014. 


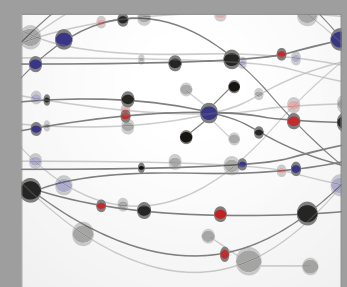

The Scientific World Journal
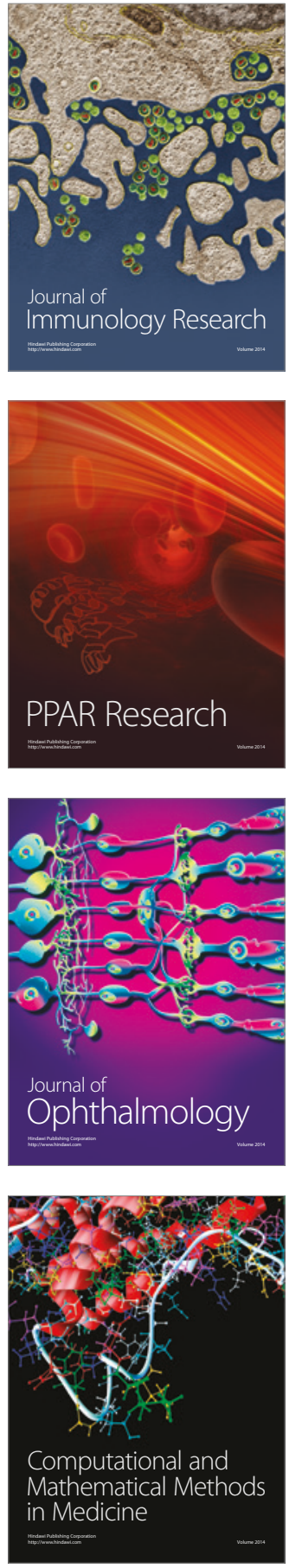

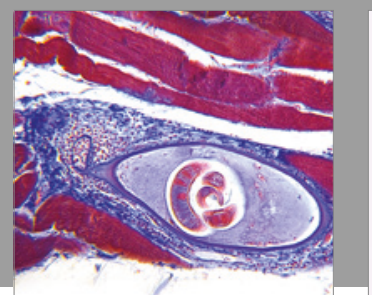

Gastroenterology Research and Practice
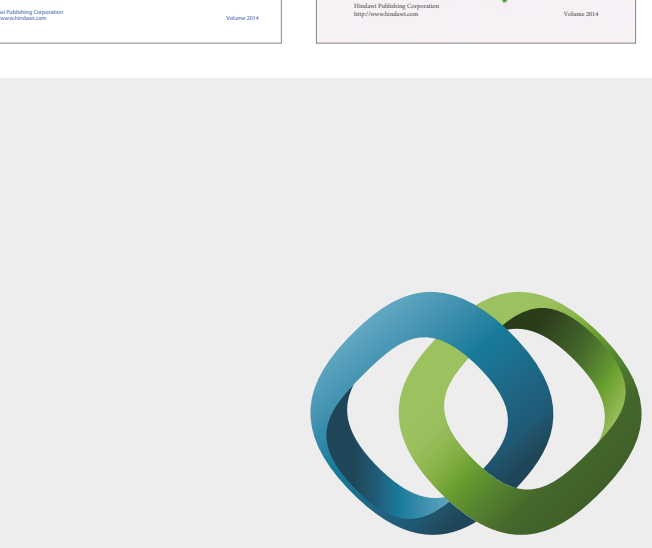

\section{Hindawi}

Submit your manuscripts at

https://www.hindawi.com
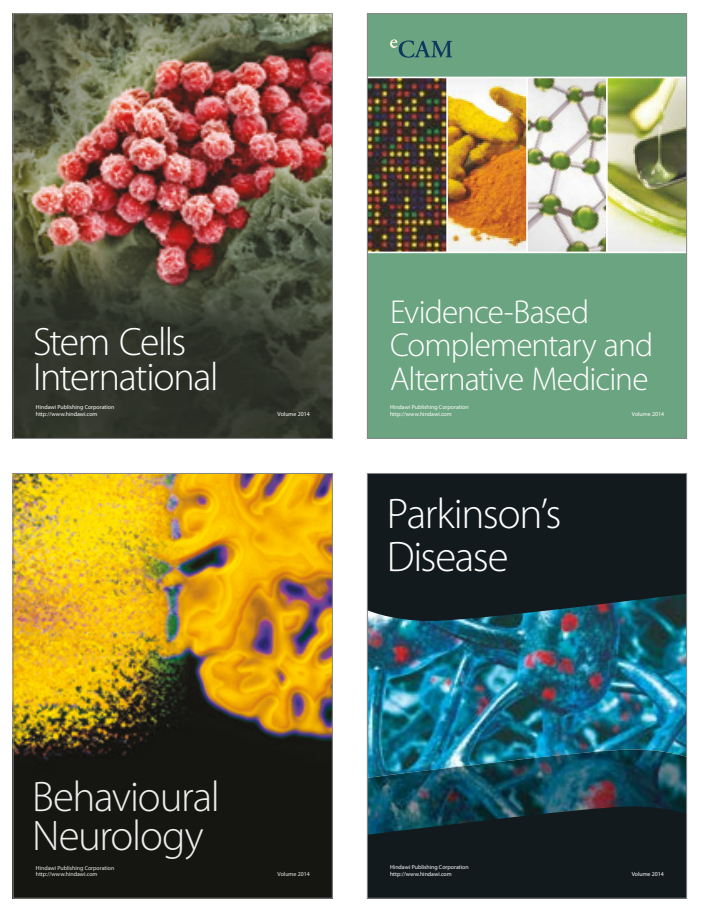
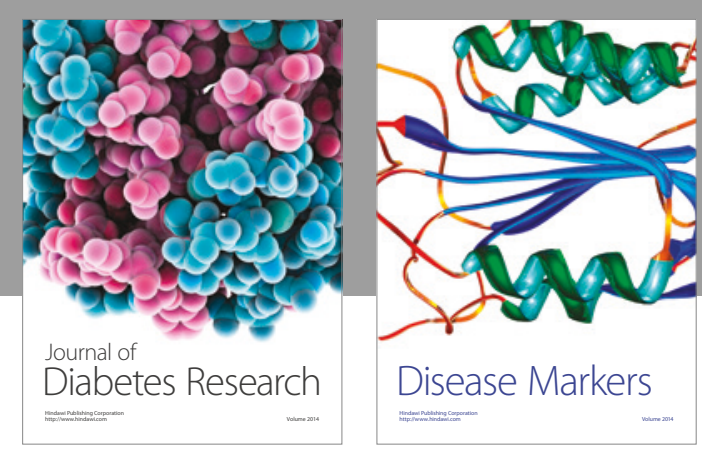

Disease Markers
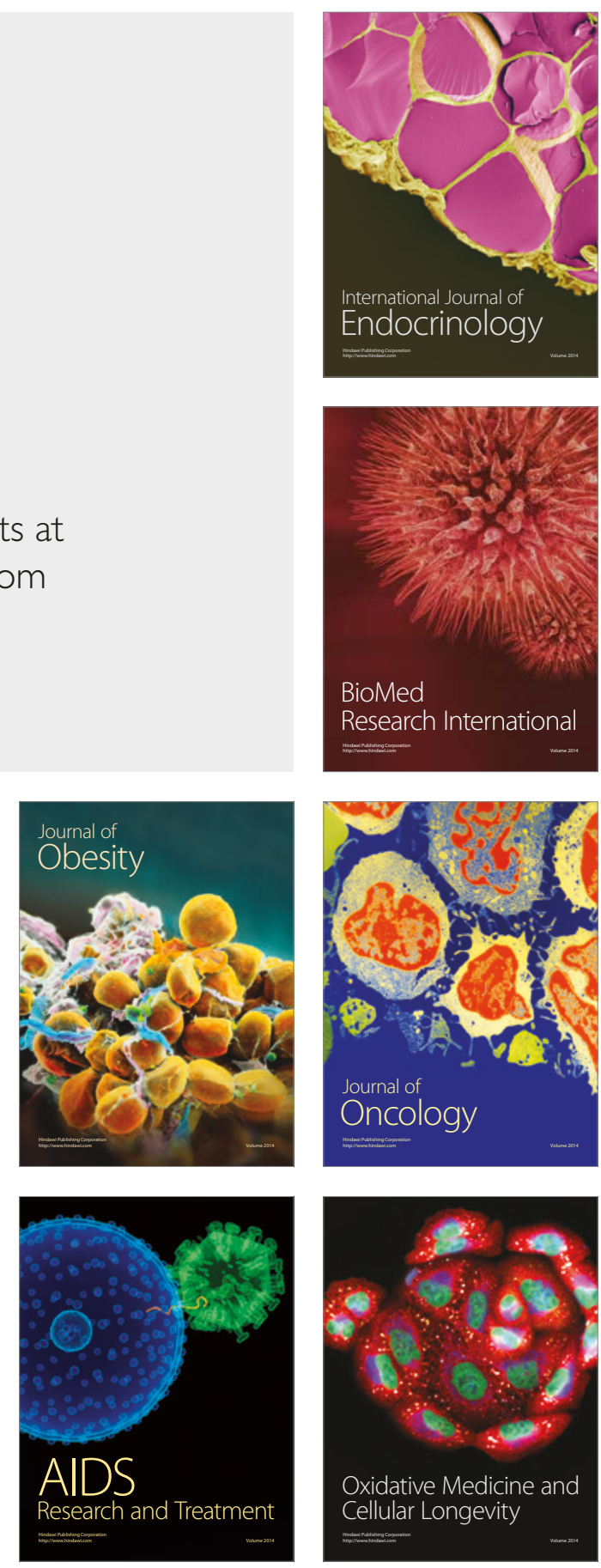